\title{
Antioxidant treatment of increased sperm DNA fragmentation: Complex combinations are not more successful
}

\author{
Cevahir Ozer \\ Department of Urology, Baskent University, Adana, Turkey.
}

\begin{abstract}
Summary Objective: Oral antioxidant supplementation is part of the treatment of infertility associated with oxidative stress-related sperm damage. It is possible to assume that the combined use of antioxidants will be better than single agent use. The purpose of this study was to compare the effectiveness of different antioxidant combinations in infertile men with increased sperm DNA fragmentation.

Materials and methods: We retrospectively reviewed the records of 637 patients who underwent antioxidant support therapy for increased sperm DNA damage between 2014 and 2019. Patients with DNA damage of $30 \%$ or more were included study.

Result: A total of 163 patients with follow-up data and who fulfilled the study criteria were included in the study. There were four different treatment groups. No statistically significant differences were found between the groups. After 3 months of antioxidant treatment, there was a statistically significant decrease in sperm DNA damage in all treatment groups. However, there was no statistically significant difference between the treatment groups.

Conclusions: The complexity of the antioxidant combination may not contribute to the success of the treatment or may cause possible side effects, increase the cost of treatment and decrease patient compliance.
\end{abstract}

KEY WORDS: Infertility; Oxidative stress; Antioxidants.

Submitted 3 August 2020; Accepted 8 September 2020

\section{INTRODUCTION}

The conventional semen analysis remains the main diagnostic tool for evaluating male factor infertility (1). However, difficulties resulting from the methodology of the conventional semen analysis significantly reduces the potential diagnostic power of this test. Furthermore, the conventional semen analysis cannot clearly identify all cases of male infertility. Although conventional semen analysis identifies some features of sperm function, it does not fully assess functional sperm competence (2). The search for a high-diagnostic method that could better predict the etiology and reproductive outcomes of male infertility resulted in a focus on sperm DNA integrity and fragmentation (3-5). The level of sperm DNA fragmentation seems to correlate negatively with pregnancy and delivery in both natural and assisted conceptions. It is also strongly associated with recurrent spontaneous abortion $(6,7)$.
Oxidative stress (OS) is one of the major causes of DNA fragmentation in spermatozoa (8). This makes antioxidants a part of treatment in male infertility. Since OS can be caused by vastly different oxidants, it is possible to assume that combination therapy will be better than a single agent in antioxidant therapy (9).

The purpose of this study was to compare the effectiveness of different antioxidant combinations in infertile men with increased sperm DNA fragmentation.

\section{Materials AND METHOdS}

\section{Patients}

We retrospectively reviewed the records of 637 patients who underwent antioxidant support therapy for increased sperm DNA damage between 2014 and 2019. The baseline clinical evaluation for each patient included a comprehensive history and a complete physical examination. Semen samples were collected after 2-7 days of sexual abstinence in a specially designated room in our embryology laboratory, with the aid of audiovisual stimulation. Conventional semen analysis was performed according to the criteria of World Health Organization (WHO). DNA fragmentation in spermatozoa was measured using the terminal

deoxyribonucleotidyl transferase-mediated dUTP nickend labelling (TUNEL) assay (Cell Death Detection Kit, Roche Biochemicals, Mannheim, Germany) according to the manufacturer's instructions with minor modifications. All semen tests were repeated at 3 months of treatment.

Blood samples were taken in the morning to measure follicle-stimulating hormone (FSH), luteinizing hormone (LH) and total testosterone levels at baseline and at the end of 3 months of antioxidant treatment.

\section{Inclusion and exclusion criteria}

Patients with DNA damage of 30\% or more were included study. Presence of varicocele, leukocytospermia, known genetic abnormality, history of chemotherapy and/or radiotherapy, history of malignancy, history of orchiectomy and/or orchiopexy and patients receiving hormonal therapy were accepted as exclusion criteria.

\section{Ethical consideration}

The study was approved by Institutional Review Board (Project no: KA19/250). 
Data interpretation and statistical analysis There were 193 patients who met the study criteria, had follow-up results and used 4 different antioxidant treatment protocols. Age, infertility period, history of varicocelectomy, cigarette smoking, alcohol consumption, serum FSH, serum LH and serum testosterone levels, initial conventional semen parameters, and sperm DNA damage rate before and after treatment were determined. We used propensity score-matched analysis to balance differences in age, duration of infertility, smoking and alcohol use among antioxidant treatment groups

Power value was $76.0 \%$ for an effect size value (0.179) that was calculated for sperm DNA fragmentation at 3 month of study. With this power value, it was found that the sample size was sufficient.

Statistical analysis was performed using the statistical package SPSS software (Version 25.0, SPSS Inc., Chicago, IL, USA). If continuous variables were normal, they were describle as the mean \pm standard deviation $[(p>0.05$ in Kolmogorov-Smirnov test or Shapira-Wilk $(\mathrm{n}<30)$ ], and if the continuous variables were not normal, they were described as the median. Comparisons between groups were made using one way ANOVA for normally distributed data and Krukal Wallis test were used for the data not normally distributed. Since analysis of variance was significant, comparisons were made using the Post Hoc test or Mann-Whitney U test. The catagorical variables between the groups was analyzed by using the Chi square test. Values of $p<0.05$ were considered statistically. G-power (Version 3.1, Department of Psychology, University of Düsseldorf, Germany) was used for post hoc power analysis.

\section{Results}

After the treatment groups are homogenized, a total of 163 patients with follow-up data and who fulfilled the study criteria were included in the study. All patients had no known medical problems. The medical history of the patients revealed varicocelectomy in 41 patients. Patient characteristics are presented in Table 1.

No statistically significant differences were found between the groups with regard to age, infertility period, smoking, alcohol consumption, history of varicocelectomy, the initial semen parameters and initial sperm DNA damage rate (Table 1 ).

There were four different treatment groups that met the criteria. Group A received $500 \mathrm{mg}$ daily vitamin C (EsterC plus, Solgar, USA), 400 IU daily vitamin E (Evicap fort, Kocak Farma, Turkey) and $600 \mathrm{mg}$ daily N-acetylcysteine (NAC) (Assist plus, Bilim, Turkey). Group B received 100 mcg daily selenium (Selenium, Solgar, USA) and $100 \mathrm{mg}$ daily coenzyme Q10 (coQ10) (Coenzyme Q-10, Solgar, USA) in addition to vitamin $C$, vitamin $E$ and NAC. Group C received commercial multiantioxidant supplement (NeoFortil M, Tani Pharma, Turkey) in addition to vitamin C, vitamin E and NAC. Group D received another commercial multiantioxidant supplement (Promotil men, Centax Pharma, Turkey) in addition to vitamin C, vitamin $\mathrm{E}$ and NAC. The multiantioxidant supplement used in Group C (NeoFortil M) contains a daily dose of $600 \mathrm{mg}$ L-Carnitine, $250 \mathrm{mg}$ L-Arginine, $120 \mathrm{mg}$ vitamin C, $72 \mathrm{mg}$ vitamin E, $15 \mathrm{mg}$ coQ10, $60 \mathrm{mcg}$ selenium, 40 $\mathrm{mg}$ zinc sulfate and $800 \mathrm{mcg}$ folic acid and the other one used in Group D (Promotil men) contains a daily dose of $2000 \mathrm{mg}$ L-carnitine, $500 \mathrm{mg}$ L-arginine, $200 \mathrm{mg}$ vitamin C, $120 \mathrm{mg}$ vitamin E, $100 \mathrm{mg}$ coQ10, 60 mcg selenium, $60 \mathrm{mg}$ magnesium, $15 \mathrm{mg}$ zinc sulfate, $400 \mathrm{mcg}$ folic acid, 2 mg vitamin B6, 6 mcg vitamin B12, 10 mcg vitamin D, $1 \mathrm{mg}$ vitamin A and $2 \mathrm{mg}$ beta-carotene.

Table 2.

The effect of oral antioxidants on sperm DNA damage.

\begin{tabular}{|lccc|}
\hline & Initial DNA damage (\%) & $\mathbf{3}^{\text {th }}$ month DNA damage $(\%)$ & $\mathbf{p}$ \\
\hline Group A & $47.72 \pm 17.29$ & $29.51 \pm 12.99$ & 0.0001 \\
\hline Group B & $50.77 \pm 11.26$ & $25.12 \pm 13.81$ & 0.0001 \\
\hline Group C & $46.71 \pm 10.84$ & $23.88 \pm 16.07$ & 0.0001 \\
\hline Group D & $51.12 \pm 18.32$ & $26.28 \pm 18.20$ & 0.0001 \\
\hline Total & $49.06 \pm 14.03$ & $26.03 \pm 14.98$ & 0.0001 \\
\hline
\end{tabular}

\section{Figure 1.}

Change in DNA damage according to treatment groups.

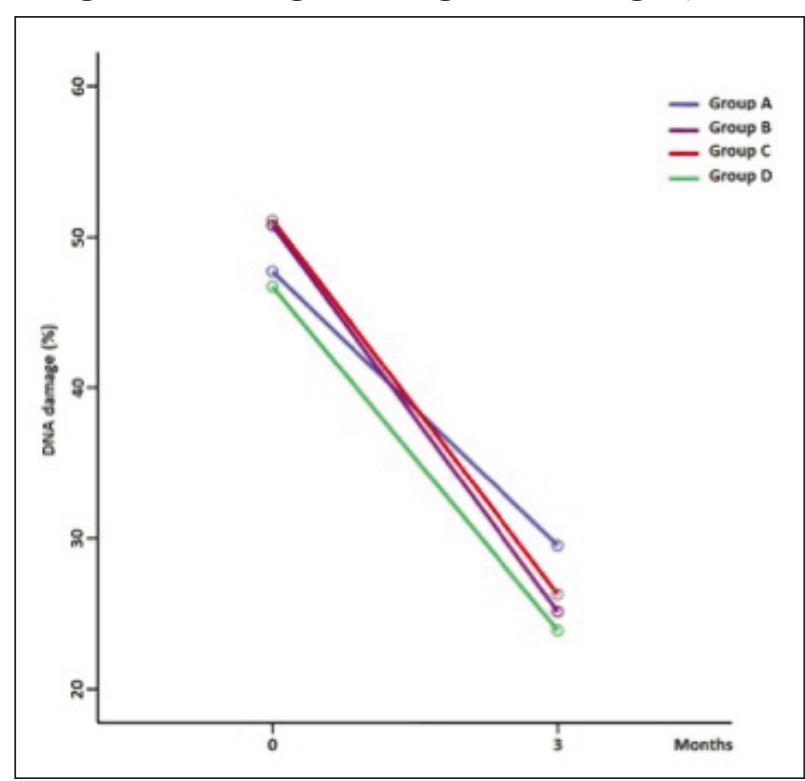


After 3 months of antioxidant treatment, there was a statistically significant decrease in sperm DNA damage in all treatment groups (Table 2 and Figure 1). However, there was no statistically significant difference between the treatment groups $(\mathrm{p}=0.230)$.

None of the patients had side effects requiring discontinuation of antioxidant support treatment.

\section{Discussion}

DNA fragmentation of spermatozoa occurs during spermatogenesis and maturation processes. Increased sperm DNA fragmentation appears to be associated with impaired sperm function. Aging, poor lifestyle-related habits (such as smoking, alcohol consumption, environmental radiation and pollution), diseases, drugs, inflammation and infection in the external genital tracts and varicocele may cause an increase in sperm DNA fragmentation $(8,10-12)$. All these factors induce sperm DNA breaks by three main mechanisms: apoptosis, impairment of sperm chromatin maturation and OS (13).

OS is a condition that is associated with an imbalance between the production and removal of reactive oxygen species (ROS) and free radicals (14). Antioxidant defense system act as scavengers to neutralize free radicals and overcome the adverse results of OS $(14,15)$. Antioxidant system including enzymatic factors (superoxide dismutase, catalase, and glutathione peroxidase), non-enzymatic factors and low-molecular weight compounds (glutathione, NAC, vitamin E, vitamin A and C, coQ10, carnitines, myo-inositol, lycopene etc.) and nutrients (selenium, zinc, and copper) can protect the body against OS (1618). Lack of one of these leads to a reduction in the antioxidant capacity of the plasma (17). The rationale behind the use of oral antioxidant therapy is that seminal OS is due to increased ROS production and/or decreased levels of enzymatic and non-enzymatic seminal antioxidants $(19,20)$. To date, several studies have shown that exogenous antioxidants have the capacity to counteract oxidative damage or OS, improving sperm DNA integrity for infertile men with OS $(18,21)$. Many oral formulations of antioxidants are readily available in the market and are commonly used to treat men with infertility. The different oral antioxidants available belong to the exogenous antioxidant category and they include Vitamin C, Vitamin E, coQ10, NAC, carnitines, trace elements such as zinc, selenium, pentoxifylline, and a combination of these oral antioxidants (20). Numerous studies have been conducted to assess the effectiveness of oral antioxidant supplementation for the treatment of infertile men with sperm DNA damage. Most of the studies showed an improvement in one or more of seminal fluid parameters, whereas some studies reported no positive effect. Although there is still no consensus on the type, dosage and duration of antioxidants to be used in the treatment, it appears that in the case of OS, doses of antioxidants should be higher than the usual daily dose and because the time required for the development of a mature sperm from spermatogonia is $72 \pm 4$ days, should be used for at least three months $(17,22,23)$.

Since OS may be due to multiple sources, it seems reasonable to assume that a combination of antioxidants tar- geting the male reproductive system will provide better protection than a single antioxidant (9). However, the complexation of antioxidant therapy may have potential adverse effects including reductive stres (21).

Furthermore, complex combinations may increase the cost of treatment and may decrease treatment compliance (24). According to our study including 163 patients, complexity of oral antioxidant combination treatment does not appear to contribute to treatment success.

The limitation of our study was its retrospective and non-randomized nature. Although the small sample size in the groups we compared is another limitation, sample size was found to be sufficient in post hoc power analysis. The variability of the compounds, especially in commercial combinations, is the weakness of our study. Since placebo arm ethics is arguable in such studies, we believe that the lack of placebo arm of the study is not a limitation. Furthermore, the effect of improvement in sperm DNA damage on fertility has not been studied because the female group was not homogenous.

\section{Conclusions}

The use of oral antioxidants can help to reduce OS and to treat OS related sperm DNA damage. The use of antioxidant agent combinations is expected to be more successful in the treatment than a single agent. However, it should be noted that the complexation of the combination may not provide an additional contribution to treatment success. Therefore, possible side effects, treatment cost and patient compliance should be kept in mind when designing combination protocols.

\section{ACKNOWLEDGEMENTS}

The author would like to thank Biostatistics specialist Cagla Sariturk for her expertise and assistance in statistical analysis.

\section{REFERENCES}

1. Komiya A, Kato T, Kawauchi Y, et al. Clinical factors associated with sperm DNA fragmentation in male patients with infertility. Scientific World Journal. 2014; 2014:868303.

2. Santi D, Spaggiari G, Simoni M. Sperm DNA fragmentation index as a promising predictive tool for male infertility diagnosis and treatment management - meta-analyses. Reprod Biomed Online. 2018; 37:315-326.

3. Bungum M. Sperm DNA integrity assessment: a new tool in diagnosis and treatment of fertility. Obstet Gynecol Int. 2012; 2012:531042.

4. Agarwal A, Said TM. Role of sperm chromatin abnormalities and DNA damage in male infertility. Hum Reprod Update. 2003; 9:331345.

5. Sakkas D, Mariethoz E, Manicardi G, et al. Origin of DNA damage in ejaculated human spermatozoa. Rev Reprod. 1999; 4:31-37.

6. Agarwal A, Cho CL, Esteves SC. Should we evaluate and treat sperm DNA fragmentation? Curr Opin Obstet Gynecol. 2016; 28:164-171.

7. Evgeni E, Charalabopoulos K, Asimakopoulos B. Human sperm 
DNA fragmentation and its correlation with conventional semen parameters. J Reprod Infertil. 2014; 15:2-14.

8. Wright $C$, Milne S, Leeson H. Sperm DNA damage caused by oxidative stress: modifiable clinical, lifestyle and nutritional factors in male infertility. Reprod Biomed Online. 2014; 28:684-703.

9. Lewis SE, John Aitken R, Conner SJ, et al. The impact of sperm DNA damage in assisted conception and beyond: recent advances in diagnosis and treatment. Reprod Biomed Online. 2013; 27:325-337.

10. Pourmasumi S, Sabeti P, Rahiminia T, et al. The etiologies of DNA abnormalities in male infertility: An assessment and review. Int J Reprod Biomed (Yazd) 2017; 15:331-344.

11. Cai T, Wagenlehner FM, Mazzoli S, et al. Semen quality in patients with Chlamydia trachomatis genital infection treated concurrently with prulifloxacin and a phytotherapeutic agent. J Androl. 2012; 33:615-623.

12. Alargkof V, Kersten L, Stanislavov R, et al. Relationships between sperm DNA integrity and bulk semen parameters in Bulgarian patients with varicocele. Arch Ital Urol Androl. 2019; 91.

13. Moustafa MH, Sharma RK, Thornton J, et al. Relationship between ROS production, apoptosis and DNA denaturation in spermatozoa from patients examined for infertility. Hum Reprod. 2004; 19:129-138.

14. Pham-Huy LA, He H, Pham-Huy C. Free radicals, antioxidants in disease and health. Int J Biomed Sci. 2008; 4:89-96.

15. Kao SH, Chao HT, Chen HW, et al. Increase of oxidative stress in human sperm with lower motility. Fertil Steril. 2008; 89:11831190.
16. Calogero AE, Condorelli RA, Russo GI, La Vignera S. Conservative nonhormonal options for the treatment of male infertility: antibiotics, anti-inflammatory drugs, and antioxidants. Biomed Res Int. 2017; 2017:4650182.

17. Walczak-Jedrzejowska R, Wolski JK, Slowikowska-Hilczer J. The role of oxidative stress and antioxidants in male fertility. Cent European J Urol. 2013; 66:60-67.

18. Arcaniolo D, Favilla V, Tiscione D, et al. Is there a place for nutritional supplements in the treatment of idiopathic male infertility? Arch Ital Urol Androl. 2014; 86:164-170.

19. Zini A, Al-Hathal N. Antioxidant therapy in male infertility: fact or fiction? Asian J Androl. 2011; 13:374-381.

20. Alahmar AT. Role of oxidative stress in male infertility: an updated review. J Hum Reprod Sci. 2019; 12:4-18.

21. Gharagozloo P, Aitken RJ. The role of sperm oxidative stress in male infertility and the significance of oral antioxidant therapy. Hum Reprod. 2011; 26:1628-1640.

22. Alahmar AT. The impact of two doses of coenzyme $Q 10$ on semen parameters and antioxidant status in men with idiopathic oligoasthenoteratozoospermia. Clin Exp Reprod Med. 2019; 46:112-118

23. Agarwal A, Nallella KP, Allamaneni SS, Said TM. Role of antioxidants in treatment of male infertility: an overview of the literature. Reprod Biomed Online. 2004; 8:616-627.

24. McDonald HP, Garg AX, Haynes RB. Interventions to enhance patient adherence to medication prescriptions: scientific review. JAMA. 2002; 288:2868-2879.

\section{Correspondence}

Cevahir Ozer, MD (Corresponding Author)

cevahirozer@gmail.com

Dadaloglu Mh Serinevler 2591 Sk No: 4/A, 01250 Yuregir,

Adana (Turkey) 